\title{
Leveraging Robotics Research for Children with Autism: A Review
}

\author{
Luthffi Idzhar Ismail • Thibault Verhoeven . \\ Joni Dambre and . Francis wyffels
}

Accepted: November 2018

\begin{abstract}
Robotics research in helping children with autism has gained increased attention in recent years. Children with autism often struggle with neurodevelopmental disorders that affect their lives particularly in communication skills, social interaction and their repetitive stereotyped behavior. In this article, we highlight previous findings on human-robot interaction for children with autism. To date, it has been claimed that child-robot interaction is a beneficial approach in helping children with autism to improve their quality of life. Based on an extensive search of the literature, we identified three major research gaps: (1) diversity in research focus, (2) bias contribution in robotics research towards specific behavior impairments in autism and, (3) effectiveness of human-robot interaction after robot-based intervention program. Therefore, this review paper shall identify and thoroughly discuss published works that address the research gaps found in this areas. This article is therefore seen as a crucial step in bridging the gap between robotics research and children with autism. The results presented could be beneficial for researchers in determining future directions for robotics research in helping children with autism.
\end{abstract}

Keywords Human-Robot Interaction - Robotics · Children with Autism

L. I. Ismail, T. Verhoeven, J. Dambre and F. wyffels

Department of Electronics and Information Systems, Faculty of Engineering and Architecture, Ghent University imec, Belgium

Tel: +32483319891

Fax: $(+32) 092643594$

email: luthffiidzharbin.ismail@ugent.be

\section{Introduction}

Autism is perceived as a wide spectrum disorder that can be defined as a pervasive neural-developmental disorder that affects how a person perceives the world and his interaction with others [1]. Children who are diagnosed with autism often see, hear and feel the world differently from others. They experience difficulties in basic communication skills, have limited social interaction with people and posses repetitive and unusual stereotyped behaviors. However, some children with autism (CWA) have a very high Intelligence Quotient (IQ) whereby some are gifted in memorizing while others may develop special mathematical talent. While autism is incurable, early intervention programs are believed to be able to help them in improving their quality of life [2-4]. Therefore, early intervention programs should be targeted at toddlers, so that they can have a better quality of life as they grow up.

CWA can normally be identified and diagnosed by using Gilliam Autism Rating Scale which is a normreferenced assessment tool that assists clinicians in identifying and diagnosing autism worldwide [5]. This assessment tool reflects the definition of autism, which characterizes autism in three behavior impairment subscales (stereotyped behavior, communication and social interaction). Each sub-scale has detailed descriptions of its characteristics. Stereotyped behavior sub-scale includes common characteristics such as lack of eye contact and repetitive unusual gesture. On the other hand, communication sub-scale characteristics manifested by CWA include unsuitable verbal and non-verbal communication. Meanwhile, in social interaction sub-scale, CWA normally portray inappropriate emotion, improper gesture and lack of eye contact in their daily interaction with their peers, teachers and family members. 
The definition and use of the term autism has greatly evolved over the past century. As people become more aware and informed about autism through its wide definition, more and more children are being diagnosed and labelled as autistic [6]. In 2013, the definition of autism has changed to include only dual impairments namely communication and social interaction [7] as opposed to the earlier definition consisting triad impairments as previously mentioned. The new definition of autism thus refers to a neuro-developmental disorder that affects an individual in social communication and repetitive stereotyped behavior.

Various reports have been published reporting on the number of children diagnosed with autism. Based on statistical data published in Morbidity and Mortality Weekly Report (Center for Disease Control and Prevention) in 2008, one in every eighty-eight children in the United State was identified as having Autism Spectrum Disorder [8]. A later report published by Center for Disease Control and Prevention on the other hand reported that in 2010, the number of children diagnosed with autism has increased to one in every sixty-eight children [9]. These reports have shown that the number of children diagnosed with autism is steadily increasing over the years.

In Europe, there are no official published resources that investigate the prevalence or statistic of CWA. However, Roelfsema et al. (2012) reported on the prevalence of autism based on three regions in the Netherlands, which are Eindhoven, Haarlem and Utrecht [10]. Based on the published results, the number of children diagnosed with autism is quite significant. Based on reported statistics, the prevalence of CWA in Eindhoven region is 229 per 10,000 followed by Haarlem (84 per 10,000) and Utrecht (57 per 10,000).

In Asia, the prevalence of children diagnosed with autism is also found to be quite substantial. In a study conducted in Ilsan district of Goyang City, South Korea, the prevalence of children diagnosed with autism was estimated to be 2.64 percent from 55,266 children population aged between $7-12$ years old [11]. The statistics were gathered based on the assessment conducted by medical experts with the consent obtained from the participants' parents and guardians. Although the report did not specify the parameters of the study in terms of its age range, method of diagnosis, and etc., the reported number of CWA indicated the prevalence of CWA in Asian countries such as South Korea.

The increased number of children diagnosed with autism is clearly evident based on the statistical report published thus far. However, the numbers of medical personnel and occupational therapists (OT) are still limited despite the increase in the number of CWA. The limited number of OT poses a challenge to cope with providing effective intervention program for CWA. It should be noted that it takes seven years to train a qualified medical doctor (depending on universities' medical program), four years to train a certified OT (depending on universities' occupational therapy degree program) and two or three years to train a certified child-care (depending on course/program). To overcome the shortage of OT, developing robotic tools for autism intervention programs are necessary in order to increase the capacity of medical trained person to provide intervention programs for CWA $[12,13]$. Without a robotic tool, one medical trained person could only manage to help two CWA at a time. However, with a robotic tool, one medical trained person could provide help to more than two CWA. To date, researchers and engineers who are involved in building social robots for CWA use available robotic tools in the market to help CWA by providing more interactive human-robot interaction during robotbased intervention program.

In this paper, we conducted an exhaustive review on the work done by other researchers in helping CWA through the use of robotics platforms. The objective of this review paper is two-fold; to acknowledge the gaps that exist in the field of robotics research as mentioned earlier and to shape the future research direction in the area. In general, despite a large number of robotics tools for CWA that have been developed, there seemed to be no standardized references or benchmark with regard to the contributions of robots towards dual major impairments in autism. In addition, robotics research for CWA are found to be lacking in terms of the reporting of the effectiveness of long-term intervention program. Therefore, this paper aims to examine and systematically analyze previous findings in these areas in order to identify potential directions for future research.

\section{Novelty of Our Review}

Various aspects with regard to robotics research for CWA have been successfully conducted and reviewed. This section describes the previous reviews of robotics research for CWA. For example, Kozima et al. (2009) presented a comprehensive survey on research, therapy and entertainment for CWA which utilized Keepon robot [14]. Ricks et al. (2010) on the other hand studied the trends and consideration in Robot-Assisted Autism Therapy [15]. In 2011, Diehl investigated the clinical use of robots for individuals with autism [16] by focusing on several aspects such as the response of CWA towards robots, the way of eliciting autistic behavior using robots and the use of robot as a model, agent or teacher and robot to give feedback or encouragement. 
Scassellati et al. (2012) also analysed various aspects in autism research which focused on physical appearance of robots, human-robot interactions, data analysis and trends in Socially Assistive Robotics (SAR) for CWA [6]. Bharatharaj et al. (2013) [17] deliberated various considerations (i.e. psychological, physiological and social interaction) in autism therapy that utilized robotics approaches while Boucenna et al. (2014) conducted a review on interactive technologies such as robotics system for CWA [18]. In the same vein, ArestiBartolome et al. (2014) reviewed various technological approaches that could help to support individuals with autism [19].

In order to assess the inclination of future medical practitioners to use robots in autism intervention, Conti et al. (2015) conducted a survey which investigated the acceptance of robotics approach for autism intervention programs among future psychology practitioners [20]. Although the survey was only limited to 74 participants (37 from Italy and 37 from United Kingdom), overall participants in this study showed a global positive attitude towards Socially Assistive Robotics (SAR) paradigms. Apart from that, some researchers also conducted studies investigating the establishment of robot-based therapeutic protocol for CWA training [21-23] and the needs of therapists towards having a robotic platform in helping CWA [24, 25].

Another review of using social robots for CWA was conducted by Pennisi et al. (2015) which focused on the usefulness of using social robots in autism therapy [26]. Peca et al. (2016) also conducted a survey to measure the ethical acceptability in robotics research for CWA [27]. The results of the study revealed that the majority of the respondents in the survey agreed with the usability of robots in robot-based intervention programs for CWA. Additionally, the results also showed that the respondents also shared their views on the enhancement of robot therapy for CWA.

It is noted that some doubts may arise on the use of robots in autism intervention programs and issues on human-robot interaction have been highlighted and discussed in [28]. Begum et al. (2016) conducted a study on the readiness of robots to perform autism intervention for special children [29]. Moreover, Coeckelberg et al. (2016) conducted a survey on the expectation and role of robot in robot-assisted therapy for CWA [30] whereby multiple issues have been discussed such as ethical acceptability, trust, sociability and appearance.

More recently, Sartorato et al. (2017) discussed on the ways researchers could improve therapeutic outcomes by enhancing social communication and sensory processing with the use of interactive robots [31]. Besides that, Broadbent (2017) deliberated on the appli- cation of social robots in human-robot interactions from the perspective of psychologists [32]. This is deemed to be essential in the development of robot technology and the study of human behavior [33]. Broadbent (2017) emphasized on how human think, feel and behave towards robots. Apart from that, a systematic review on the intervention for CWA targeting on sensory difficulties has also been made by Weitlauf et al. (2017) [34].

Based on previous reviews, we conclude that one can identify three major gaps in robotics research: (1) lack of diversity in research focus, (2) bias contribution in robotics research towards specific behavior impairments in autism and, (3) effectiveness of human-robot interaction after robot-based intervention program. These aspects are seen to be very crucial as these are the areas that can help to bridge the gap that currently exists in literature and in return help to shape future works that can be conducted particularly in the area or robotics intervention for CWA.

In the following sections we elaborate more on these gaps which is important for researchers in shaping the future work in robotics intervention for CWA. The remainder of this review is structured as follows: Section 3 discusses the review process and how we screened more than 400 research articles based on three important parameters which are (A) research that utilized physical robot, (B) articles that contain medical and engineering content, $(\mathrm{C})$ research that have at least one contribution towards three major impairments in autism. In section 4, we discuss the experimental setup of previous work include the physical robots that has been used, intervention duration, interaction setting and diagnosis method in their studies. Then, section 5 discusses the research focus of previous work in detail. Next, Section 6 elaborates on other contributions towards autism impairments. Moreover, section 7 discusses the intervention effectiveness utilizing robot as a tool in previous studies. Lastly, section 8 discusses on future research direction which are expected to close some of the research gaps found in literature. This is very important for researchers in shaping the future work in robotics intervention for CWA.

\section{Method of Review}

Over the last decade, many articles in robotics research for CWA have been published, varying from education to entertainment, design to diagnosis and from therapy to training [35-42]. Some researchers provide more focus on engineering perspective such as robots design, robots' controller, user-friendly programming platform and robots' kinematics [38, 43-48]. Meanwhile, other researchers gave more attention to medical points of view 


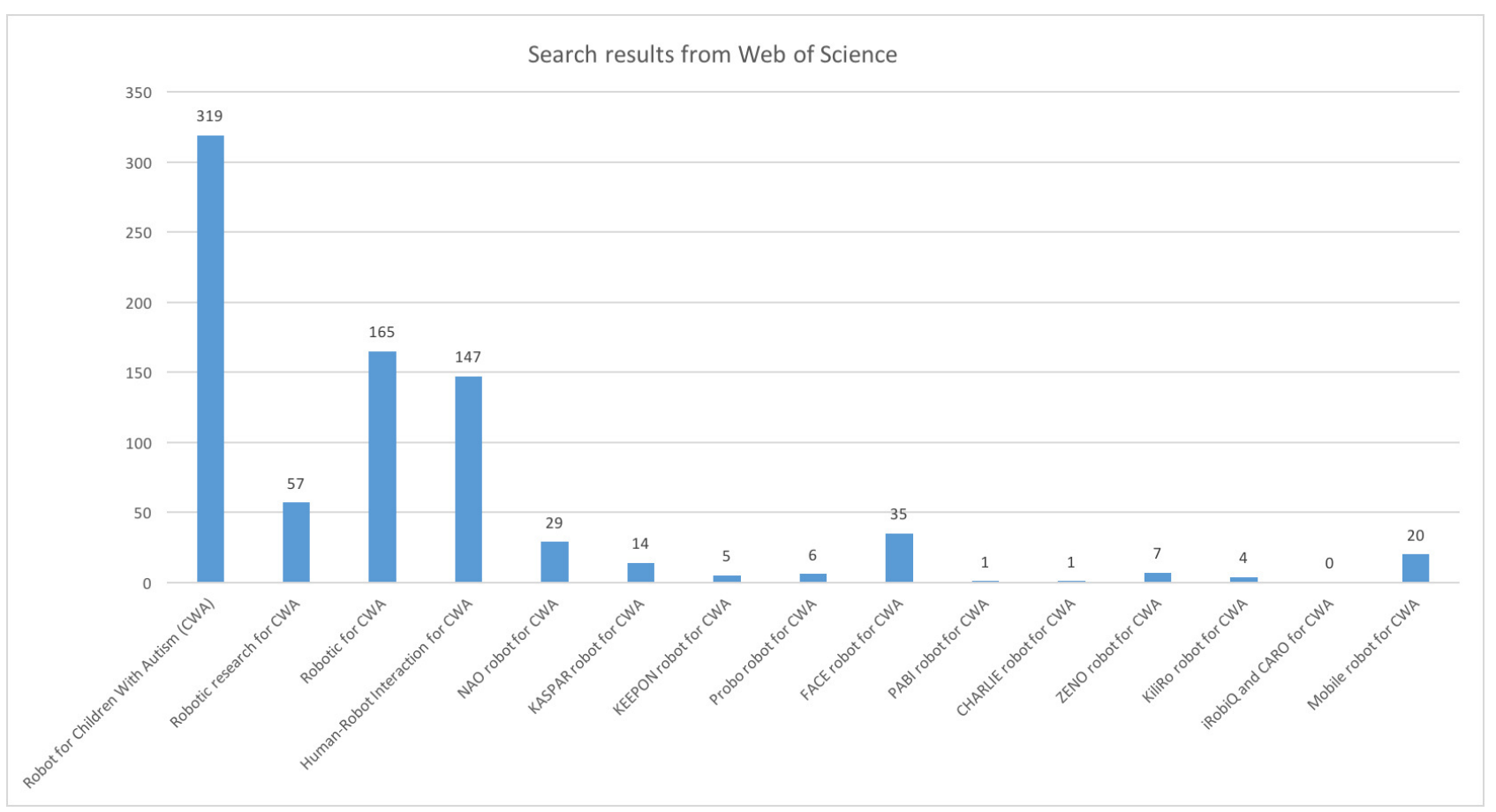

Fig. 1: This figure shows the number of articles and journals from web of science. Research articles was searched and reviewed based on certain keywords such as 'robotic research for children with autism (CWA) as discussed in section 3 .

where they provide further elaboration demographic of participants (such as diagnosis, age range, gender, experimental protocol and behavioral analysis [49-54].

In our study, research articles were selected based on certain parameters in the Web of Science website. Specific key words were used for our article search including robot for CWA, Robotics Research for CWA, Robotic for CWA, Human-Robot Interaction for CWA, NAO robot for CWA and etc. Figure 1 illustrates the results from the Web of Science search.

Based on these findings, the search was then continued with the robots' name and certain parameters that will be explained in the review. Furthermore, all selected papers selected for the review were published from the year 1900 to February 2018. To iterate, research on robotics for autism only vigorously started in early 21th-Century. However, definition of autism has been established in early 20th-century [1] while human-robot interaction was established in the early 19th-Century. More than 400 published papers have been downloaded, reviewed and analysed for the purpose of this article.

In order to filter more than 400 papers, we defined three main parameters. These three parameters were set in order to determine the direction for future research based on collected works of previous researchers in the area. Based on the three parameters, articles must:

A : have physical robots;

$\mathrm{B}$ : contain medical and engineering content;

C : have at least one contribution towards three major impairments in autism.

Using the parameters set to narrow down the scope of the review, we found forty-one papers that matched with our review criteria (complying with items A, B and $\mathrm{C}$ ). Those papers are tabulated in Table 1 for further discussion in the next sections. Apart from papers that are discussed in Table I. It should be acknowledged that there are many other autism interventions studies which also used robots in their research. However, these papers do not fit with our review criteria and therefore excluded from Table 1. Nevertheless, their research contributions are significant to the field of robotics research for CWA and therefore are aptly referred to and acknowledged throughout this article.

\subsection{Robots}

First and foremost, as previously mentioned, we only reviewed research papers that used physical robots for intervention programs or as therapeutic tool for CWA. 
It was evident that some researchers used virtual robots in helping CWA as they are reported to bring several benefits to CWAA $[55,56]$. However, this review paper takes into account only published works that optimized physical robots for child-robot interaction. Table I shows the list of robots that were used in robotics research for CWA.

\subsection{Medical and Engineering Content}

Robotics research for CWA is known as an interdisciplinary research field between medical and engineering disciplines. As discussed in the previous section, there were some articles that focused on the engineering content such as the design and development of the robots, the mechanical and electrical components of the robot and etc. There were also some papers that focused on the clinical perspective. In this review, we narrowed down our search to include articles that mention both, a physical robot and medical information about CWA. Based on literature [57-59], these parameters are found to be crucial particularly for future experimental setup, design of research methodology, and selection of participants as advised by clinical physicians. The medical information includes the following details:

- Duration of the intervention

- Setting of intervention experiment

- Number of children with autism

- Main inclusion criteria

- Age range

- Diagnosis method

\subsection{Contribution Towards Three Major Impairments} in Autism

To recapitulate, the review is only limited to papers that specify contributions made towards specific impairments. To recap, autism is regarded as a brain developmental disorder that affects an individual in having a stereotyped behavior, communication difficulties and struggles in social interaction. This review paper is limited to assessing articles that mention at least one of the following contributions towards three impairments which are:

- Communication

- Social Interaction

- Stereotyped Behavior

As described in the previous section, in 2013, the definition of autism has been revised from previously being defined as having triad impairments to a more recent definition of autism which includes only dual impairments [7]. Following the revised definition, two subscales which include social interaction deficits and lack of communication skills have been merged as one subscale of impairment labelled as social communication difficulties. In Table 1, we maintained the three subscales of autism impairments despite the recent definition of autism as consisting dual impairments as there were some papers that were published before 2013 that contributed to the social interaction cluster and not to the communication cluster $[60,61]$. For papers that were published after 2013, we labeled 'yes' for both communication and social interaction columns if the papers address any contributions in social communication impairments of CWA and vice versa.

\section{Review on Experimental Setup of Previous Work}

\subsection{Robots}

Thus far, humanoid robots have been successfully used and implemented in robotic intervention program for CWA. It this section, we reviewed some of the most significant works on robots that assisted CWA. Review of robots in this section were made based on sequence presented in Table 1. The most famous robot that has been widely used in robotic intervention programs for CWA is the humanoid robot NAO as shown in Figure $2(\mathrm{a})$. The humanoid robot NAO is very fascinating and currently being used in many research groups to help CWA [62-66]. Now is a programmable and a flexible robot with its 25 Degree of Freedom (DoF). NAO's physical appearance is very appealing to children as it resembles the features of a small child and is also attentive to children [67].

Technically, NAO is equipped with many sensors such as, Force Sensitive Resistors, Sonars, Inertial Units, joint position sensors, tactile sensors, 2 cameras, 4 microphones, and Light Emitting Diodes. NAO is empowered by a Lithium ion battery and can be sustained for 60 minutes in active mode. There are quite a number of researchers who currently use the humanoid robot NAO in helping CWA [62-66]. However, only nineteen published papers are found to thoroughly describe the use of NAO that fit with our three main review criteria.

The humanoid robot KASPAR is another robot that was found to be beneficial to CWA. KASPAR was invented at University of Hertfordshire, United Kingdom and developed by a group of researchers lead by Professor Kerstin Dautenhahn in 1998. Basically, KASPAR is a small and expressive humanoid robot as shown in Figure 2(b). One of the striking features of KASPAR 
is its child-like appearance. This robot is designed to function as a therapeutic toy for CWA [68]. The KASPAR project was launched in 2005 and has been successfully used in many play scenarios and games in order to socially engage child-robot interaction. Technically, KASPAR has 17 DoF and has the capability to produce facial expression, perform some gestures and make some upper body movements. KASPAR is also equipped with a camera on each eye. Moreover, tactile sensors are attached to the robots face, feet, chest and arms. With these sensors, the effects of touch interaction between child and robot can be investigated. The KASPAR robot can also be controlled through a computer or a remote control. In our review, six research articles that used KASPAR robot met our three main criteria of review and are discussed in the next section.

In Japan, Kozima and Nakagawa (2006) developed the Keepon robot in their effort to help CWA [69]. Three papers that utilized Keepon robot in their robotic research for CWA have been identified in our study. The Keepon robot consists of 4 DoF. This robot was designed to facilitate the social development of CWA. Keepon can be empowered by batteries or an electrical adapter. Its features include having multi-axis movements, built-in microphones, invisible touch sensors, and is able to listen to music. Keepon has a very attractive feature with its simple appearance in yellow color as shown in Figure 2(c). Since October 2011, Keepon is commercially available throughout the United States, United Kingdom and Europe.

Very few robots have been built and developed to cater for the complexities of human's feeling and emotion. Based on our review, we found a research developed by a group of researcher in University of Pisa, Italy that built a humanoid robot FACE (Facial Automation for Conveying Emotions). FACE is capable of expressing and conveying emotions as shown in Figure 2(d). In social interaction, expression of emotion is very important. Consistent with the challenges faced in developing a robot that fulfills the complex nature of human's feelings and emotions, only two published works were found that fit our review criteria which used humanoid robot FACE in their robotics research for CWA.

Besides humanoid robots, zoomorphic robots were found to be associated with robotics research for CWA. An example of this is PROBO (Figure 2(e)), a huggable social robot developed in Belgium [70]. The most dominant feature of PROBO is its movable trunk that resembles an aardvark and is also equipped with a screen to interact with. Its head can be fully actuated. PROBO has 20 DoF that was designed to encourage facial expression, gestures and speech for CWA. There were three published works that have been found utilizing
PROBO robot in helping CWA and are further discuss in the next section.

Another robot that has been used in robotic research for CWA is known as the CHARLIE robot. CHARLIE was designed by doctoral students from University of South Carolina, United States of America. CHARLIE is equipped with a camera that is installed in CHARLIE's nose as shown in Figure 2(f). In our review, only one research article reported on utilizing that used CHARLIE as their robotic tool in their robot-based intervention program for CWA. Other robots have also been found to be utilized and implemented in robotics research for CWA. These include Zeno robot, cuDDler robot, KiliRo, iRobiQ and CARO robot also have been used in previous robotics research for CWA as shown in Figures 2(g), 2(h), 2(i) and 2(j) respectively [71, 72].

It has been known that dynamic interaction could be established using a mobile robot. However, mobile robots could also be harmful to CWA compared to robots in static positions. Most children tend to engage physical contact with a robot which provide excellent opportunities for communication and social interaction. However, if there is contact with the robot's parts (such as robots' joints), it can cause severe injuries to children.

In our assessment, we found that most of the robots remained in a static position during human-robot interaction. This was partly due to the robots' built-in capabilities. For instance, KASPAR, FACE, PROBO, Keepon and CHARLIE were designed only for upper body movement [70, 73-75]. However, their upper body movement is sufficient to create an interesting interaction between human and robot. On the other hand, humanoid robot NAO was designed not only to move back and forth but is also capable of turning left and right. However, our review indicated that researchers who used humanoid robot NAO in their studies did not take full advantage of these features. This could be due to some ethical issues in the physical contact between child-robot that could harm the children due to the motor's stiffness or because they would like to keep the human-robot interaction simple [76, 77]. It could also be due to the fact that the robot's walking gait is dissimilar to humans. This can cause CWA to imitate the robot's walking gait which would not be a good example for children's motor development. Additionally, children might also be frightened if the robot starts to move aimlessly. Thus, simple interaction can sometimes be a better approach in maintaining children's focus and attention during human-robot interaction.

Despite the claims made on the harmful effects of using mobile robots, there are a few researchers who utilized mobile robot in their studies for child-robot in- 
teraction in autism intervention. Goulart et al. (2014), for instance, used mobile robots as a robotics tools for child-robot interaction [78]. One advantage of using a mobile robot is its ability to detect CWA for childlocalization thus ensuring a safe distance between children and robots. Moreover, the distance between children and robots can be measured and used as an indicator of children's interest towards robots as compared to human.

\subsection{Duration of Intervention}

In conventional human-human interaction, therapists and medical experts usually provide a series of intervention programs for CWA depending on the condition of patients, conventional intervention program (such as speech therapy, joint attention therapy, imitation game, cognitive module) and other behavioral training [79]. Intervention for CWA is never easy and takes a lot of time. Normally, tailor-made therapy activities in conventional intervention programs will take at least 25 hours per week [80]. Following this, improvement in their behavior will be observed by medical experts from time to time and their activities will be adjusted according to their behavioral performance.

Based on our review, most robotics intervention programs for CWA did not meet this standard as a comparison. In most robotics research for autism, robots were introduced to CWA for a short-term intervention $[63,70,81-88]$. In addition, some researchers only present their robot to the children for a one-time interaction while others would do so for several more times. In interdisciplinary research, there are many challenges faced when preparing the human subjects and experiment preparations. Furthermore, developing a suitable robotics platform for medical application would require more time.

Due to time constraint, it is understandable that most researchers only conducted a single pilot study [89-91] or short-term study to validate and proof any concept, preliminary observation or initial results [92]. It should be noted that human-robot interaction will not be beneficial and successful if the duration of interaction between robots and CWA is limited. The duration of the robotic intervention program has to be atleast equal to the minimum duration of human-human interaction. Some researchers are well informed about these issue. Anamaria et al. (2013) conducted a longterm robotics intervention program for CWA [93]. Moreover, Kozima (2005 and 2007) and his team also conducted long-term human robot interaction program for CWA [61, 94]. More researchers who conducted long- term studies in human-robot interaction for CWA are presented in Table 1.

Besides the duration of the robotic intervention program for CWA, the duration of interactions with the robot in every robotics intervention session also plays a significant role. Based on input from certified therapists, long interactions between robot and CWA would cause children to become restless, bored and lose their concentration. On the other hand, interactions that are too short will not be sufficient for children to establish eye contact and to be able to attract their attention. Therefore, optimal duration needs to be determined for optimum interaction between CWA and robots. Some researchers designed 30 minutes interaction for CWA and their respective robot. To date, there are no standardized guidelines that have been endorsed by any medical scientific committee to propose the optimal duration of interaction and the duration of robotic therapy program for CWA. Short term and long term observation of robotic intervention programs are important aspects in ensuring that the children get sufficient exposure to the robot in the aspect of behavior improvement.

\subsection{Human-Robot Interaction Setting}

The location and experiment setting for CWA to interact with a robot are important. For simple human-robot interactions, a robot can be placed in a room or classroom where CWA normally perform their daily activities. This can take place in their classroom in schools, or at any autism care centers. When researchers introduce a robot to them, the challenges faced also need to be taken into consideration. In robotics, a smart robot will need a smart environment to be fully programmed and functional. Robots need to be programmed to their known environment in order to operate in autonomous ways. Otherwise, the robot needs to be controlled wirelessly or remotely. Therefore, researchers need to adapt the environment of the robot along with the environment of CWA.

However, for more advanced interactions that involve mobility and movement, object tracking and recognition, face tracking and recognition as well as object drop and pick-up, the environment has to be known to the robot and has to be familiar to CWA. This proves to be a challenge as robots are typically programmed based on the laboratory lighting and environments. When robots are brought to the classroom, sometimes it could not function as planned. Alternatively, if CWA are brought to the laboratory, the laboratory environment may be new to them. This will 


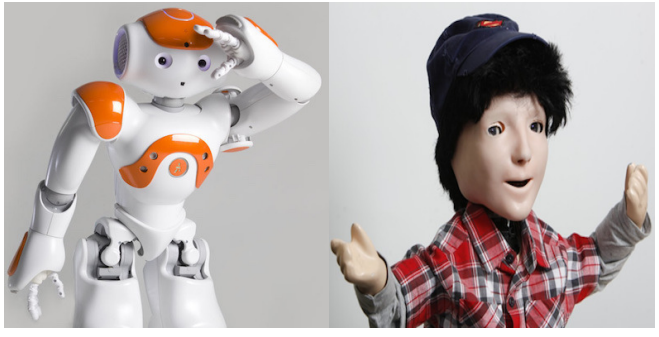

(a) NAO

(b) KASPAR

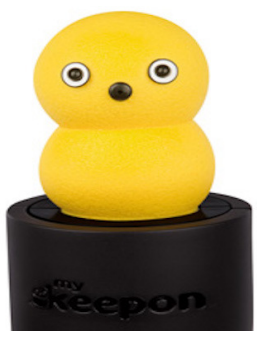

(c) Keepon

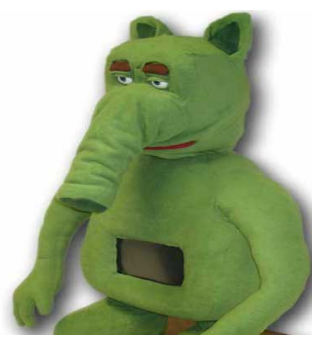

(e) PROBO

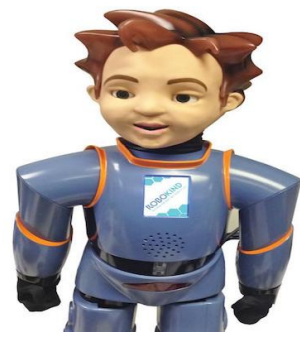

(g) ZENO

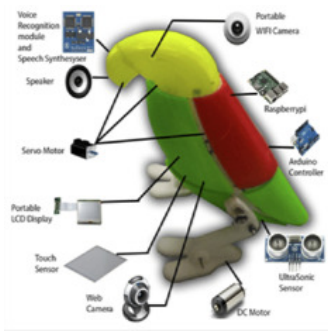

(i) KiliRo

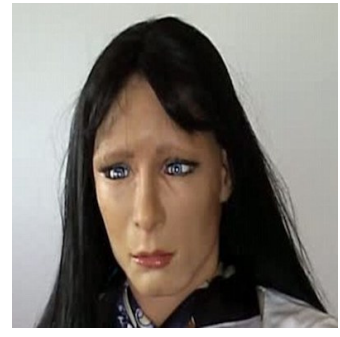

(d) FACE

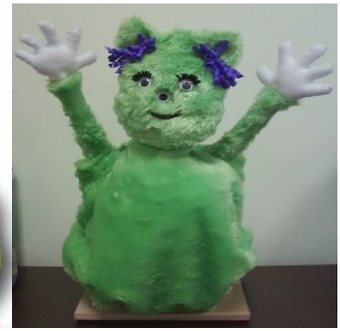

(f) CHARLIE

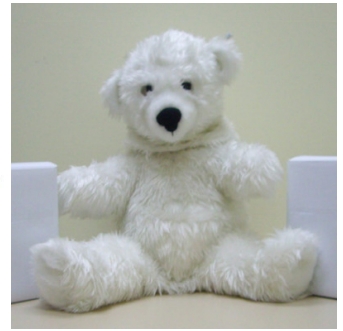

(h) CuDDler

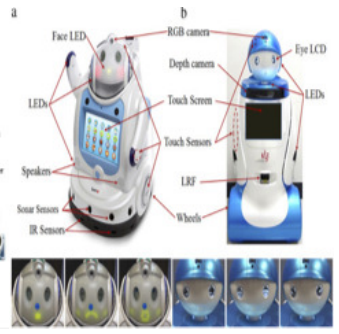

(j) iRobiQ and CARO

Fig. 2: a) Humanoid robot NAO, b) Humanoid robot KASPAR, c) Humanoid robot Keepon, d) Humanoid robot FACE e) PROBO robot , f) Humanoid robot CHARLIE , g) Zeno robot, h) CuDDler robot i) KiliRo robot j) iRobiQ (left) and CARO (right) robot

inevitably cause the experiment results to be biased to the context in which it takes place.

In this review, many researchers conducted their tests and experiments in classroom which are located in schools and autism centers $[60,61,68,83,94,95]$. This helps the CWA since they are familiar with the environment. However, the interaction with the robot will only involve a simple interaction. To have a complex robot interaction, the robot needs recognizable environment. Usually, the environment in a classroom at schools and autism centers have certain limitations in terms of limited space, back light, and other facilities. Further limitations are experienced when children come from different schools or autism centers. This will pose difficulties for researchers to setup an experiment setting in multiple schools or autism centers. Moreover, the results also can be biased since different schools have different types of facilities. Thus, the results may become non-comparable despite the CWA having the same diagnosis.

For some researchers, a special room was setup in order to conduct their experiments [85, 86]. Some used designated laboratories located in hospitals or therapy center for their experiment [63, 70, 82, 84, 87, 88, 93, 96] while some researchers did not provide any details regarding their experimental setup as shown in Table 1. With a standardized special room for experimental purposes, the robot can be programmed for more complex interactions with autistic children. Moreover, a wider range of children diagnosed with autism from various schools and different autism centers can participate in robotic intervention programs. They could visit the laboratory room on a regular basis in order to make themselves familiar with the room before being introduced to the robots. In this way, the results obtained would not be biased to particular children from different schools and autism centers. However, many of the researchers argued that budget and limited access to hospital (room availability to convert to special room) are their main constraints.

\subsection{Demographics of Participants}

Under normal circumstances, participants often provide reliable information that ensures the reliability and validity of the research findings. However, this is not the case when dealing with CWA as participants in a study as we need to take into account issues regarding privacy and confidentiality. Another aspect that needs to be considered is the cost incurred to the study in order for a honorarium to be given to each participant. In this review, we found that researchers utilized all participants that were available to them. We also found 
that researchers made sure that consent was obtained from participants' parents, guardian or family members prior to conducting their study. Most researchers only managed to obtain less than ten CWA as their participants as presented in Table 1. However, some researchers managed to obtain more than ten participants $[63,81,84]$. Alternatively, some researchers also used Typically Developed (TD) children to participate in their study as a control group $[68,96]$.

Children diagnosed with autism are categorized in a very wide spectrum. To manage this, most researchers include specific inclusion criteria of autism in their study. Among the main inclusion criteria reported in literature are mild autism, severe autism, high-functioning autism and low-functioning autism. Acknowledging these criteria is an important step before deciding on the type of human to robot interaction to be used. Different categories of autistic children require specific kind of interaction. For instance, severe autistic children cannot participate in a tactile interaction that is designed for mild autism as children with severe autism react very aggressively when interacting with a robot. A more suitable interaction for children with severe autism is a nonphysical interaction. In our review, we noted that some researchers draw a limitation line based on several main inclusion criteria of Autism Spectrum Disorder (ASD) as shown in Table I. However, some researchers did not mention any specific inclusion criteria for their participants $[60,63,64,66,82-84,95-97]$. This could be due to the confidential and privacy issues that have been agreed between researchers and guardian of CWA concerning confidentiality and privacy issues.

Since there is no specific medication that can cure autism, early intervention is crucial in order to facilitate CWA to function as close as possible to normal children. Therefore, early intervention is really crucial [98-100]. In our review, many researchers intended to provide a robotic intervention program for children aged below 15 as shown in Table I. There are some researchers who also performed their research for individuals with autism until the age of 20 [86] while some researchers conducted experiments with future therapists and professionals on the robot-therapy protocol and training implementation $[23,24]$. This is also important in establishing a standardized protocol or standard operating procedure for robotics platform in autism intervention programs [21, 51, 101-104].

\subsection{Diagnosis Method}

Autism diagnosis is an important step in identifying children that struggle with Autism Spectrum Disorder (ASD). This is also important in recognizing the severity of autism through the autism characteristic observation. In this review, a majority of researchers implemented conventional method in diagnosing CWA. Various assessment tools have been used by many researchers in their study $[51,105,106]$. One of assessment tools used is the Autism Diagnosis Observation Schedule which is a standardized behavioral analysis tool that is widely used by medical experts. Certified therapists or medical trained person manually observing the behavior of children suspected to have ASD. For example, Barakova and her team (2015) used the Diagnostic and Statistical Manual of Mental Disorders in their diagnosis method [64]. In contrast, Syamimi and her team (2012) was assisted by certified Occupational Therapists (OT) and physicians during the diagnosis procedure [89]. Some researchers also investigated the IQ of CWA and relate it with their autism characteristic and behavior performance [107] while some researchers did not specify about their diagnosis method in their articles as presented in Table 1 .

In our review, we were also trying to seek research articles that use an autonomous video processing for autism diagnosis. In order to develop an adaptive and autonomous human-robot interaction, the robotics platform must include a close loop autonomous system in the diagnosis, intervention and assessment stages. However, it is very difficult to provide an adaptive and autonomous robotic system for intervention program if we do not have an autonomous diagnosis method for CWA. Monitoring and evaluating human behavior for behavioral diagnosis is a very challenging task since human behaviors are very subjective and vary from one person to another.

\section{Review on Research Focus of Previous Work}

In the previous section, we provided detailed discussion on the various robots that have been used in robotics research for helping CWA. Based on the review of literature, we found that previous works conducted have focused on providing explanations regarding participants' diagnosis and the main inclusion criteria of CWA. Researchers were also found to design their robot-based intervention program based on capabilities of the robot and its suitability to the target group of CWA. 


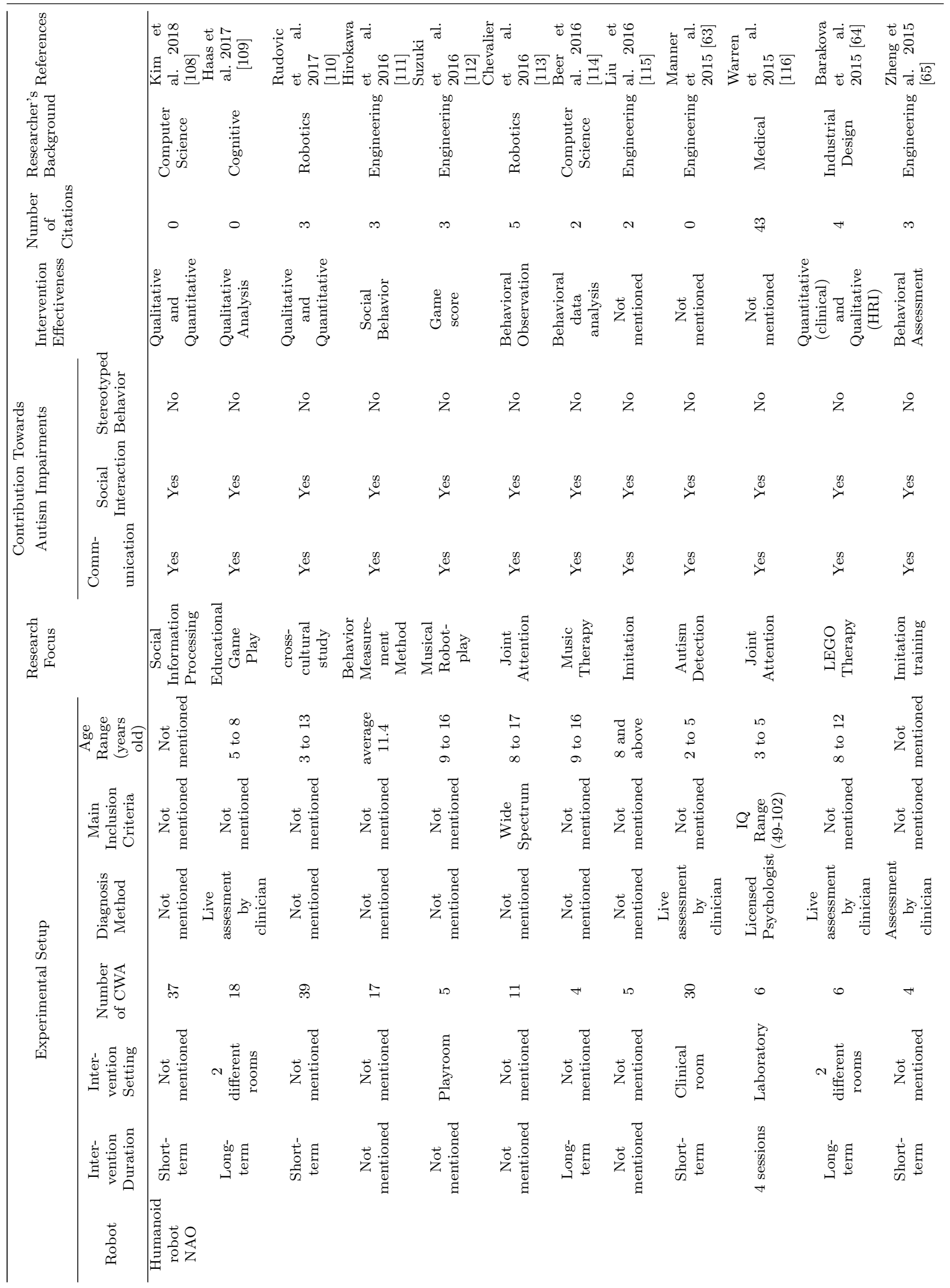




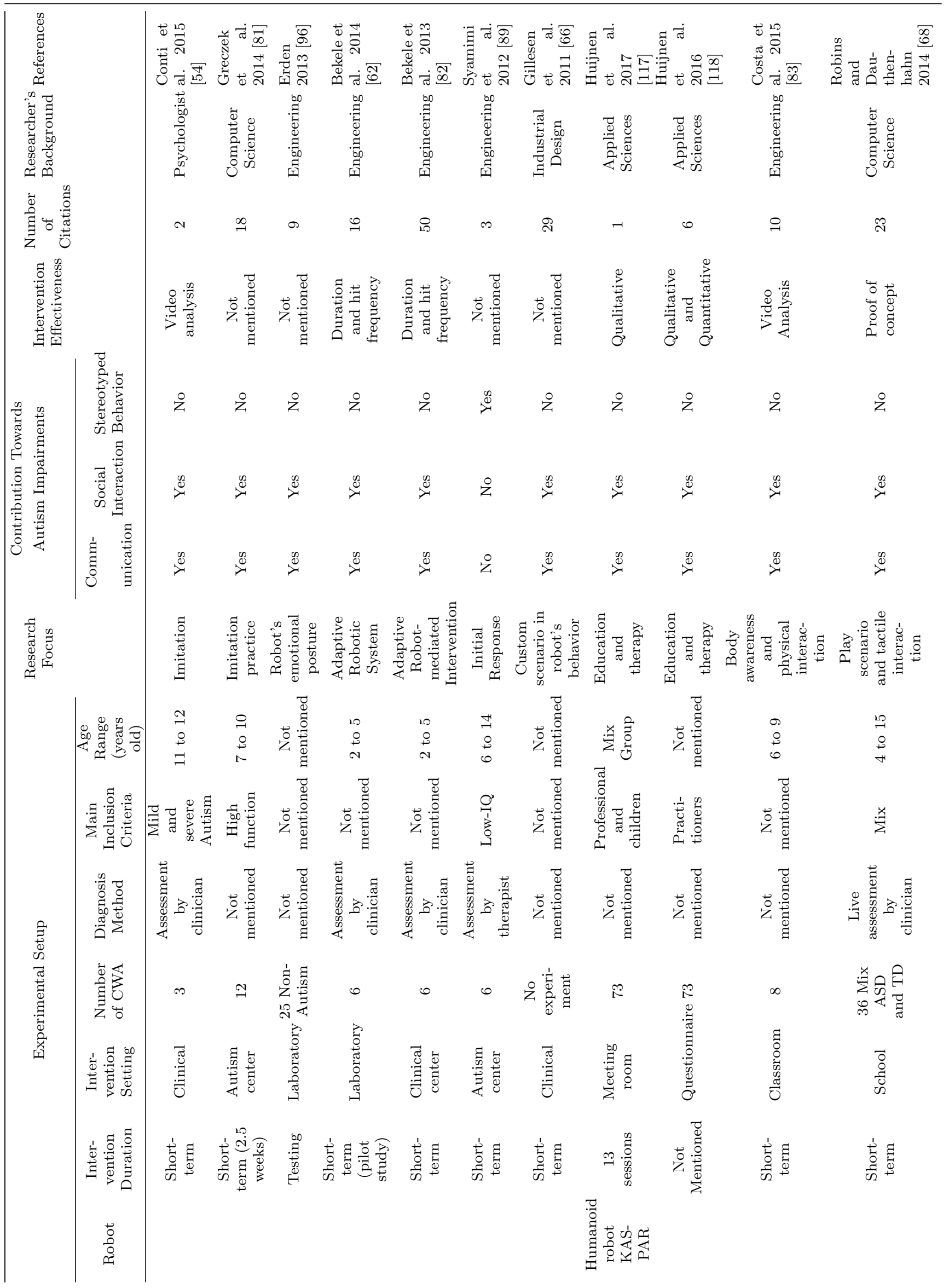




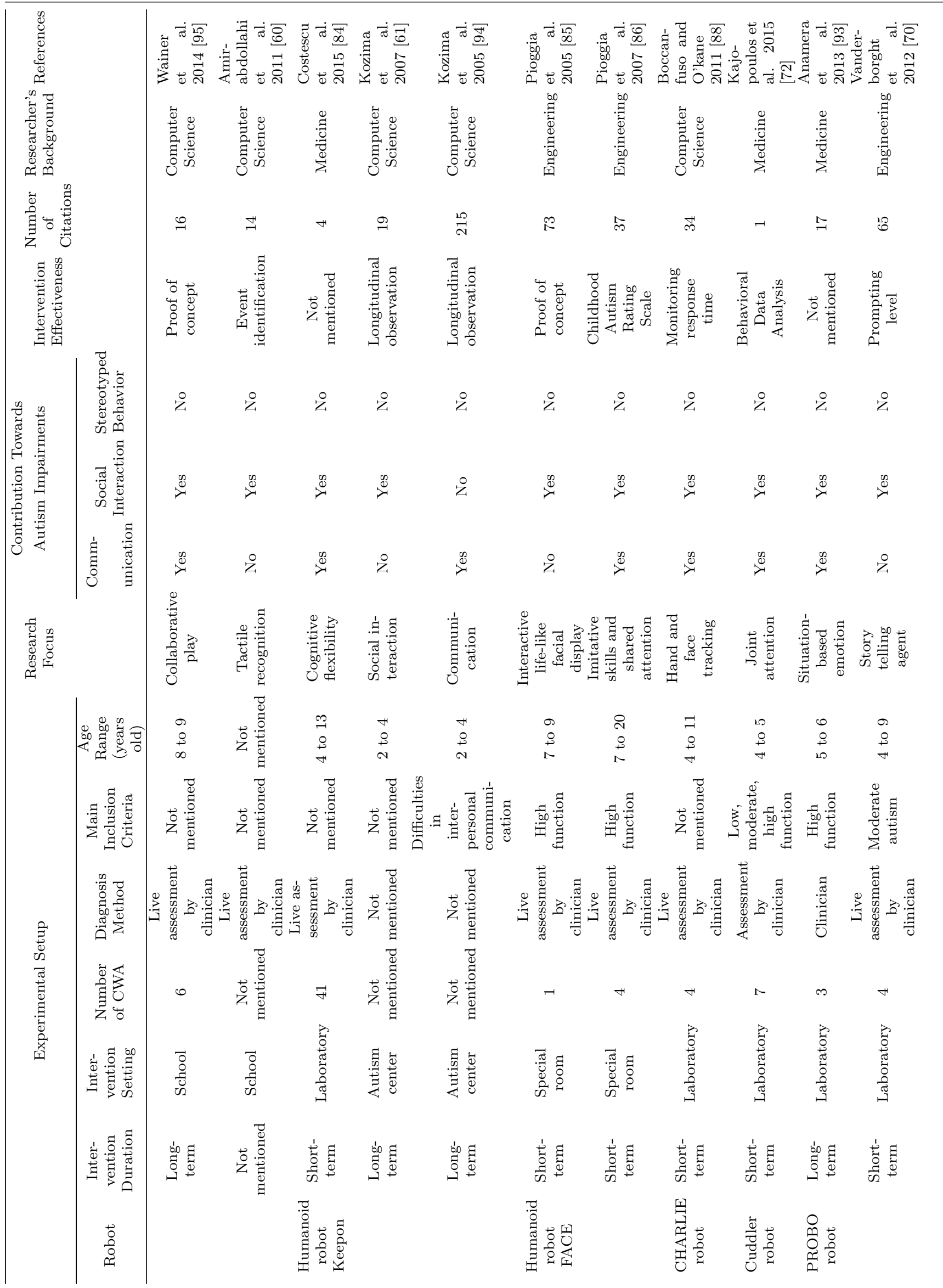




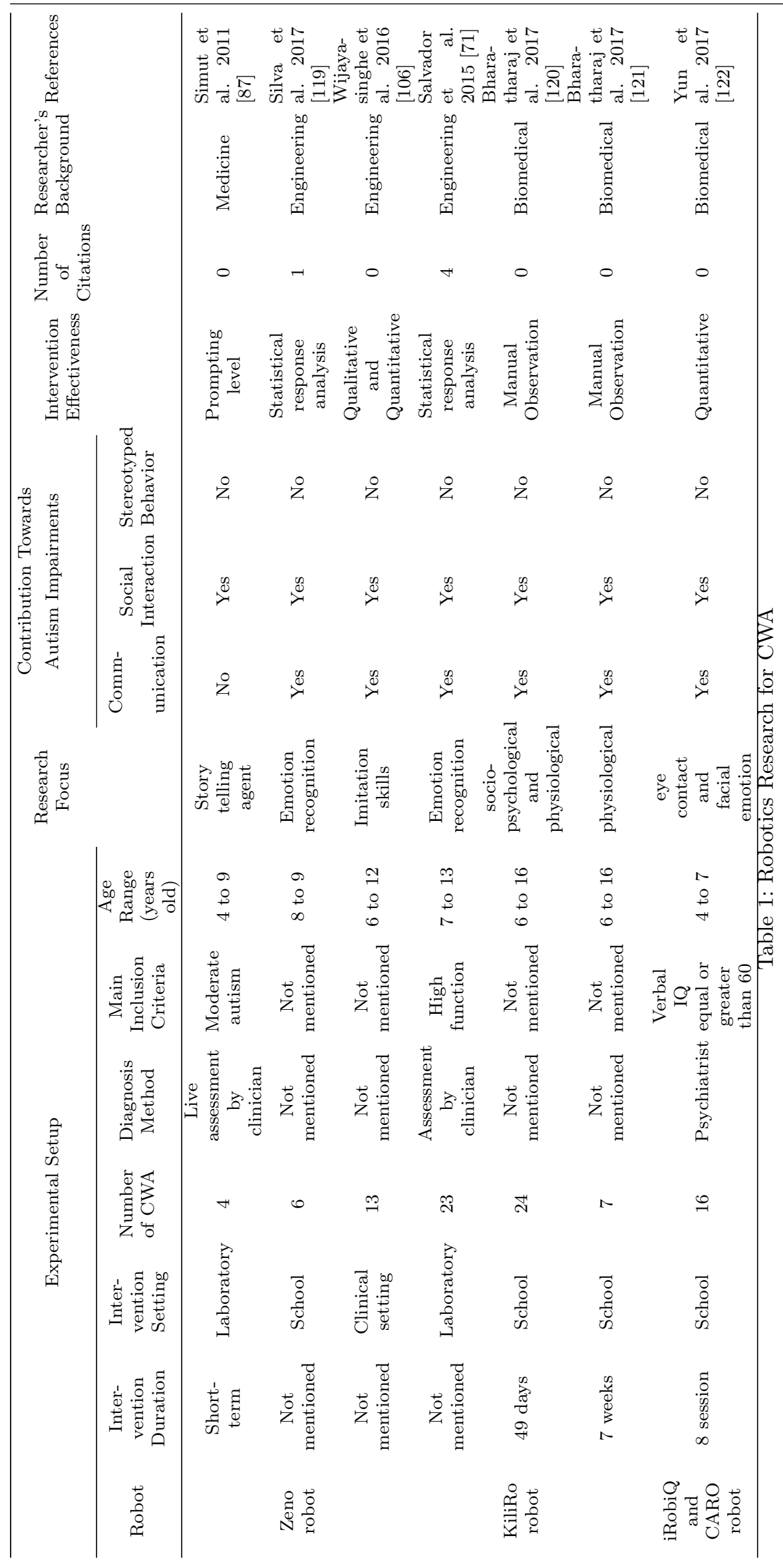


For example, many researchers focused their study on the improvement of imitation skills using their robot in order to help CWA with motor problem [54, 65, 81, 115, 123]. Moreover, Greczek et al. (2014) designed robot-based intervention program that focused on imitation practice for high- functioning CWA [81]. The humanoid robot NAO has been utilized to be the robotic tool in their study. Through imitation practice, CWA were expected to improve some aspect of their communication and social interaction skills.

Besides that, many researchers focused their robotics research for CWA who were classified as high-functioning group. This group of children basically has less severe symptoms and characteristics as compared to other categories of CWA. They usually have difficulties in language usage (i.e. humor and tolerance in give and take situation or conversation) and language delays, lack of skills in social interaction, delay in motor skills, difficulties in understanding abstract concepts and are obsessed with certain information. Research for highfunctioning group of CWA was also conducted by Pioggia and his team (2005) using humanoid robot FACE. He focused on interactive life-like facial display in 2005 [85] Later in year 2007, he then focused his research on imitative skills and shared attention for high-functioning CWA [86].

In 2013, PROBO was used for situation based emotion recognition for children with high-functioning autism by Anamaria (2013) [93], while Simut (2011) and Vanderborght (2012) used PROBO as a Story Telling Agent for children with moderate autism [70, 87]. Robins and Dautenhahn (2014) on the other hand tested their robotbased intervention program for mixed group of CWA. They focused on play scenario and tactile interaction between KASPAR robot and CWA [68]. Syamimi and her team (2012) investigated the initial response of CWA with low-IQ using NAO [89]. Kozima et al. (2005) focused their research in communication for CWA that have difficulties in interpersonal communication [94].

As pointed out earlier, some researchers did not clearly mentioned their target group inclusion criteria. Thus, we were unable to relate their research focus with their subjects. It could be due to the research Non-Disclosure Agreement (NDA) or for the purpose of protecting the privacy of CWA and family. Sometimes, there were also robot-based intervention program that targets CWA at a larger scale. Therefore, generalization of human-robot interaction were made for pilot study for large scale of CWA. This was necessary to identify the general patterns of responses towards some general or basic human-robot interactions including verbal and non-verbal interactions and two-way communication between robot and children. Some basic human- robot interaction was also tested for typically developed (TD) children for purpose of comparative study for intervention program that was designed for CWA [68]. It was also used to validate the robot-based intervention program that was designed for CWA. The system was normally tested to TD children before being implemented to CWA.

\section{Review on Contribution Towards Three Major Autism Impairments of Previous Work}

Many robotics for CWA researchers promised results in helping children diagnosed with autism. Contribution towards the actual needs of CWA was identified based on capabilities of the robots and the target behavior improvement in CWA. Some researchers asserted their contributions towards the improvement of communication aspects while some focused on the enhancement of social interaction skills and only few focused on reducing stereotype behavior in CWA. An acknowledgement of these contributions is crucial in order to determine directions for future research as our concern is not about the capability of the robot, but rather about how a robot can help CWA.

In our study, most researchers who used NAO as their robotic tools focused their contributions towards the improvement of communication and social interaction as thoroughly presented in the previous sections. Manner et al. (2015) designed their robotic research for CWA in improving social interaction deficit using humanoid robot NAO as their tool [63]. Barakova and her team (2015) contributed in terms of communication and social interaction by developing LEGO therapy for CWA [64]. Robotic works from Greczek and her colleagues (2014) for CWA also has contributed to the improvement of communication and social interaction through imitation practice [81].

Besides that, Bekele and his team (2013) worked on an adaptive robot mediated intervention which had contributed to the social and communication progress of CWA [82]. Erden and his team (2013) focused their contribution on social interaction impairments in CWA [96]. On the contrary, while many researchers focused their contribution on dual impairments (communication and social interaction), Shamimi and her team (2012) designed human-robot interaction module to reduce stereotyped behaviors of CWA [89]. Moreover, KASPAR has been used by researchers in the United Kingdom who contributed to improvement in autism impairments. Costa et al. (2015) reported their contribution towards communication and social interaction improvements through body awareness and physical interaction between KASPAR and CWA [83]. 
Robins and Dautenhahn (2014) also stated their contribution towards enhancements of communication and social interaction in CWA [68]. Meanwhile, Wainer et al. (2014) and Amirabdollahian et al. (2011) reported their contributions in social interaction improvement for CWA [60, 95]. Furthermore, Kozima et al. (2005) focused on communication development for CWA by using humanoid robot Keepon [94] and made further contributions in social interaction impairments in 2007 [61]. Costescu and her team (2015) also utilized humanoid robot Keepon in their contribution towards social interaction development between small children with robot [84]. Therefore, we noted that in general, robotic research for CWA has to be carried out with a specific aim and should be a goal-oriented research as a simple humanrobot interaction for CWA could hugely impact them in various ways depending on the specified aim and purpose of the research.

Social interaction difficulties are common impairments found in children diagnosed with autism. Difficulties in recognizing emotion, having inappropriate gesture movements and lacking of eye contact are among common characteristics portrayed by CWA thus indicating the challenges faced with regard to social interaction problems. This is one of the reasons why most robotics researchers for autism focused their research on social interaction problems. Pioggia and his team (2005) developed humanoid robot FACE in their effort to contribute in social interaction problem [85]. They focused on interactive life-like facial display in 2005 and further worked on the imitative skills and shared attention problem in 2007 [86].

Moreover, Simut et al. (2011) and Vanderborght et al. (2012) also focused their research in helping CWA in the area of social interaction [70, 87]. They used non-humanoid robot PROBO as a story telling agent to encourage social interaction between PROBO robot and CWA. Emotion recognition was one of the features that was developed in PROBO. Anamaria et al. (2013) studied situation-based emotion recognition [93] which contributed to the improvement of impairment among CWA in communication and social interaction. Besides that, human gesture is one of the important elements in communication skills and social interaction. Boccanfuso and Okane (2011) focused their robotics research in hand and face tracking [88]. Their research focusing on these impairments have benefited CWA and provided suggestive results to other researchers to work on this area.

As a conclusion, it is evident that many researchers focused their contributions in communication and social interaction area while very few researchers focused on how to reduce stereotyped behavior among CWA.
Nevertheless, some researchers split their research findings into separate articles to help clarify their experimental results and make their contribution to robotics research appear more substantial. This is done by separating discussions on the communication aspect in one paper and discussions on the experimental results concerning social interaction in another paper. This practice is acceptable since human behavior is subjective in nature and that their responses in human-robot interaction could be explored from different angles and perspectives (i.e communication, social interaction and . stereotyped behavior).

\section{Review on Intervention Effectiveness of Previous Work}

Most researchers claimed that robot-based intervention program is beneficial to CWA. In practice, researchers used the behavior measurement scale to analyze behavior of CWA during human-robot interaction. Some execute it in real time while others used post video analysis approach through recorded videos.

It is very difficult to measure the performance of human behaviors in a human-robot interaction. Begum et al. (2015) argued for the informative standards in human-robot interaction metrics [124] as human behaviors are subjective. Barakova and her team (2015) analyzed the impact of robot training in clinical perspective (quantitatively) and human-robot inter- action perspective (qualitatively) [64]. Bekele and his colleagues (2013) measure the human-robot interaction effectiveness based on hit frequency (frequency of looking to target) and duration of CWA looking at their robots [82].

Costa and her research team (2015) conducted a video analysis in their effort of measuring the intervention effectiveness for CWA [83]. Their data on children identifying body parts of a robot were obtained from recorded videos capturing CWA's active and tactile interaction session with KASPAR robot. The results indicated that CWA spent more time looking at the robot than the human experimenter.

Besides that, some researchers investigated their humanrobot interaction from the perspective of proof of concept [68, 95]. Robins and Dautenhahn (2014) developed a play scenario and tactile interaction between KASPAR robot and CWA while Wainer et al. (2014) developed collaborative plays between KASPAR robot and CWA [68]. Amirabdollahian et al. (2011) on the other hand had measured their human robot interaction between KASPAR and CWA by using event identification [60]. Touch events were recorded using tactile sensors recorder and automatic identification of touch events have a high degree of accuracy. 
Moreover, Kozima et al. (2005; 2007) presented their robotic research for CWA and emphasized on interactive robot for communication care [61, 94]. However, they only presented the longitudinal observation on human robot interaction without providing details on the intervention effectiveness of their longitudinal observation. Pioggia and his team $(2005 ; 2007)$ presented on their proof-of-concept of interactive life-like facial display $[85,86]$. In 2007, they used the Childhood Autism Rating Scale (CARS) for behavioral assessment in their human-robot interaction platform. Simut and Vanderborght $(2011 ; 2012)$ measured the effectiveness of their human robot interaction experiment by using prompt level approaches $[70,87]$. CWA were asked to interact with a PROBO robot and the level of prompt was measured based on their responses using a 7-point scale [70].

Boccanfuso and Okane (2011) posit that the effectiveness of an intervention is measured in time response [88]. In their study, CWA were monitored in terms of time response when they were asked to interact with a CHARLIE robot. Aspect regarding, interaction time, lost face time, passive time, active time, time of face detection and time for hand detection were recorded during the interaction between the CHARLIE robot and CWA.

Based on literature that presented on the effectiveness of robot-based intervention programs, we noted that many researchers report on short-term intervention effectiveness [68, 85, 95]. Results obtained in this area will be beneficial to the robotic researchers community as they can assist in continuing their robotics work for CWA. However, there were also some papers which did not presented the results on the effectiveness of the robot-based intervention programs conducted. This could be due to the confidentiality status of their research. Furthermore, some literature did not highlight on the long term analysis of the effectiveness of HRI algorithm in robot-based intervention programs. In view of this, researchers need to take into account regarding this issue and start implementing the long-term intervention effectiveness monitoring in robot-based intervention programs.

We conclude that long term studies and assessments are needed in order to convince people in the medical profession as well as the autism community on the effectiveness of robot-based intervention programs for CWA. At the moment, robotics research for CWA has gained much attention from many organizations such as research centers, NGOs, universities, hospitals and the ministry of health. Therefore, more long-term robotic intervention effectiveness studies need to be conducted in order to provide concrete evidence that robotic technology is indeed beneficial for CWA.

\section{Discussion of Future Research Direction}

Results from existing robotics studies were comprehensively discussed in previous section. In order to use robotics as a (therapeutic) tool to improve (one of) the Autism Behavior Impairments, research should bridge the gaps between (1) lack of diversity in research focus, (2) bias contribution in robotics research towards specific behavior impairments in autism and, (3) effectiveness of human-robot interaction after robot-based intervention program. In this section we would like to highlight the three main parameters that are crucial in shaping the future research directions.

\subsection{Future Research Focus}

In recent years, robotic research has become very prominent which see the growing number of robotic works have been done to help CWA. Various studies reported on various aspects of robotic research for CWA including play- scenario, tactile interaction, imitation, eye contact detection, story telling agent, head turning, turn taking game, emotion recognition, collaborative play, situation-based recognition, hand tracking and face tracking. Consequently, many CWA have benefited from these programs and have improved their social communication in human-robot interaction activities as mentioned in the literature.

In many studies, the research focus was tailor-made to the need of CWA. For example, Chevalier et al. (2016) conducted their study on the joint attention in order to help the target group of CWA to improve their joint attention skills [113]. A robot was used in their research to encourage CWA to improve their joint attention skills. Besides that, Suzuki et al. (2016) focused on musical-robot play in their experiment in order to improve social communication skills of CWA [112]. By merging music therapy and robotics platform, CWA became more playful as reported in their results.

Besides fulfilling the needs of the CWA, robot's design and capabilities could limit the content of humanrobot interaction. Ideally, a robot's design and its function should work as we desired. However, it is not easy to develop a robot that can function close to human or is robust to everything. Every robots has their own limitations. Thus, some researchers focus their research on the development of robotic platform $[125,126]$. Robots need to be ready while technical problems should be solved before robots can be introduced to CWA as a therapeutic tool.

Although using commercialized robot platforms is useful, they will limit researchers in solving problems beyond robot's capabilities. As an example, NAO robot 
has an appealing outlook and is capable of accomplishing various tasks. NAO is programmable and is equipped with a lot of sensors. CWA was reported to have a good initial response towards this robot as reported in previous studies [127]. However, in terms of facial and emotion expressions, NAO could have posed some limitations due to its simple outlook and facial appearance. To overcome these limitations, researchers thus need robots that can perform some tasks that can fulfill the needs of CWA.

In the future, more robots should be built based on the needs of CWA or based on the goal of the study (i.e: facial expression, emotion recognition, motor impairments). By creating more robots, therapists and CWA will get more benefits and that will make human-robot interaction more interesting and beneficial to the target group. For instance, social robot OPSORO can be customized in terms of software and mechanical design for facial expressions and emotions recognition. Besides that, the cost of building an OPSORO is also relatively cheap as compared to building a high-end robot [128]. Therapists can also play their role in providing some advice to be technical teams on the needs of CWA. Their knowledge and expertise on identifying the needs of CWA in intervention program could facilitate engineers to develop more robots and its functionalities that can assist CWA to improve their quality of life.

\subsection{Contribution towards Dual Impairments in Autism}

In terms of the contribution towards dual impairments in autism, future research aim at improving dual impairments of CWA. This means that their social communication should be enhanced with the presence of a robot in their intervention program. With the use of robots, intervention programs for CWA may become more interactive and attractive. In addition, the content of human-robot interaction should be more adaptive towards the need of CWA. Robots should be used to train CWA in improving their imitation skills, help them to improve level of eye-contact and educate them to improve their daily social skills such as verbal and non-verbal communication. Various approached can be used in order to help CWA such as 'use of robots as education-mediator', or 'use of robots to play game' or 'use of robots to be social partner'.

Moreover, future approach in human-robot interaction should be able to encourage CWA to portray less stereotyped behavior. Through the feeling of amazement on the capabilities of the robot, CWA are expected to focus into the robot activities while simultaneously ignoring their repetitive stereotyped behavior. A robot could be a positive agent in human to robot interaction. A robot is sometimes capable of attracting the focus of CWA from their repetitive stereotyped behavior. Thus, CWA will benefit from the human-robot interaction.

\subsection{Intervention Effectiveness}

Many studies focused on case-study, proof of concept, initial response, pilot study and short term behavioral assessments as reported in the previous section. However, more studies need to be conducted on a long-term basis in order to prove that robotics research could really help CWA. Therefore, researchers should plan for a long-term intervention effectiveness assessment in future robot-based intervention programs.

Systematic schedule for robot based intervention programs should be implemented when dealing with autistic children. CWA have to familiarized themselves with both the experimental setup as well as the robots. This is essential to avoid biasness in the experimental results since CWA will act differently in an unfamiliar environment. Therefore, interactions between a robot and CWA should be planned on in a regular basis for a long period of time. Assessment of behavioral response in human robot interaction should be done prior, during and after the interaction between CWA and robot.

As for the long term intervention effectiveness, monthly report should be made based on feedback obtained from each human-robot interaction and also the feedback from their parents or guardian at home. Robot-based intervention program was reported to benefit CWA. However, their actual behavior at home was not taken into account in previous studied. Thus, behavioral report on their behavior at home without a robot could also helps researchers in their future studies.

\section{Conclusion}

In the past decade, there has been a lot of research towards human-robot interaction for the benefit of children with autism (CWA). As far as the robotics research for CWA is concerned, there is a lot of research in this domain. In this review, we analyzed more than 400 papers with respect to the use of physical robots, their medical and engineering content as well their effectiveness in contributing towards one of the three major autism impairments. Based on our analysis, we find three major important future research aspects for human-robot interactions studies for CWA: (1) diversity in research focus, (2) contribution towards autism impairments and, (3) intervention effectiveness. We identified that, even though the use of robots in helping 
CWA was reported to be promising. There are still many research gaps that should be overcome as discussed in this article.

Consequently, in the next juncture, more research should focus on the content of interaction between robot and CWA. It should be based on the needs of the CWA and capabilities of the robots. Future design of humanrobot interaction should at least consist of some humanrobot activities that could contribute to the dual impairments aspect in autism. It shall encourage CWA to be better in their social communication skills as well as improving their behavior by reducing their repetitive and stereotyped behavior. Furthermore, robot-based intervention program should be planned for a short-term observation and long-term intervention effectiveness. The robot must be introduced to CWA in a systematic schedule and proper follow-up needs to be planned for longterm assessment and behavior evaluation. Improvement of behavior could be achieved and monitored through repetitive intervention program between robot and CWA.

To conclude, it is therefore believed that this exhaustive review on the published works related to robotics research for CWA provide useful insights on previous works in various aspects of human-robot interaction in improving dual impairments in CWA. It is also our hope that the review allows future researchers to identify potential areas that can be explored in hope of bridging the gap that exists in robotics research in helping CWA.

\section{Compliance with Ethical Standards}

Conflict of Interest: The authors declare that they have no conflict of interest.

\section{References}

1. American-Psychiatric-Association, Diagnostic and statistical manual of mental disorders $D S M-I V$ (American Psychiatric Association, Washington, DC, USA, 1995)

2. C.M. Corsello, Infants \& Young Children 18(2), $74(2005)$

3. A.G. Pour, A. Taheri, M. Alemi, A. Meghdari, International Journal of Social Robotics pp. 1-20 (2018)

4. S.E. Mengoni, K. Irvine, D. Thakur, G. Barton, K. Dautenhahn, K. Guldberg, B. Robins, D. Wellsted, S. Sharma, BMJ open 7(6), e017376 (2017)

5. J. Gilliam, GARS-3: Gilliam Autism Rating Scale-Third Edition (Pro-Ed Publishers Austin, 2014)

6. B. Scassellati, H. Admoni, M. Mataric, Annual review of biomedical engineering 14, 275 (2012)
7. J.C. McPartland, B. Reichow, F.R. Volkmar, Journal of the American Academy of Child \& Adolescent Psychiatry 51(4), 368 (2012)

8. J. Baio, Centers for Disease Control and Prevention (2012)

9. D.M.N.S.Y. Developmental, .P. Investigators, et al., Morbidity and mortality weekly report. Surveillance summaries (Washington, DC: 2002) 63(2), 1 (2014)

10. M.T. Roelfsema, R.A. Hoekstra, C. Allison, S. Wheelwright, C. Brayne, F.E. Matthews, S. Baron-Cohen, Journal of autism and developmental disorders 42(5), 734 (2012)

11. Y.S. Kim, B.L. Leventhal, Y.J. Koh, E. Fombonne, E. Laska, E.C. Lim, K.A. Cheon, S.J. Kim, Y.K. Kim, H. Lee, et al., American Journal of Psychiatry (2011)

12. S. Silva, F. Soares, S. Costa, A.P. Pereira, F. Moreira, in Bioengineering (ENBENG), 2012 IEEE 2nd Portuguese Meeting in (IEEE, 2012), pp. 1-4

13. J. Wainer, E. Ferrari, K. Dautenhahn, B. Robins, Personal and Ubiquitous Computing 14(5), 445 (2010)

14. H. Kozima, M.P. Michalowski, C. Nakagawa, International Journal of Social Robotics 1(1), 3 (2009)

15. D.J. Ricks, M.B. Colton, in Robotics and Automation (ICRA), 2010 IEEE International Conference on (IEEE, 2010), pp. 4354-4359

16. J.J. Diehl, L.M. Schmitt, M. Villano, C.R. Crowell, Research in Autism Spectrum Disorders 6(1), 249 (2012)

17. J. Bharatharaj, S.S. Kumar, in Computing, Communications and Networking Technologies (ICCCNT), 2013 Fourth International Conference on (IEEE, 2013), pp. 1-5

18. S. Boucenna, A. Narzisi, E. Tilmont, F. Muratori, G. Pioggia, D. Cohen, M. Chetouani, Cognitive Computation 6(4), 722 (2014)

19. N. Aresti-Bartolome, B. Garcia-Zapirain, International journal of environmental research and public health 11(8), 7767 (2014)

20. D. Conti, A. Cattani, S. Di Nuovo, A. Di Nuovo, in Robot and Human Interactive Communication (RO-MAN), 2015 24th IEEE International Symposium on (IEEE, 2015), pp. 555-560

21. S.M. Mavadati, H. Feng, M. Salvador, S. Silver, A. Gutierrez, M.H. Mahoor, in Robot and Human Interactive Communication (RO-MAN), 2016 25th IEEE International Symposium on (IEEE, 2016), pp. 855-860

22. Z. Zheng, Q. Fu, H. Zhao, A.R. Swanson, A.S. Weitlauf, Z.E. Warren, N. Sarkar, IEEE Transac- 
tions on Neural Systems and Rehabilitation Engineering 25(6), 668 (2017)

23. C.A. Huijnen, M.A. Lexis, R. Jansens, L.P. de Witte, Journal of autism and developmental disorders 47(10), 3079 (2017)

24. I. Zubrycki, G. Granosik, International Journal of Social Robotics 8(4), 553 (2016)

25. D. Conti, S. Di Nuovo, S. Buono, A. Di Nuovo, International Journal of Social Robotics 9(1), 51 (2017)

26. P. Pennisi, A. Tonacci, G. Tartarisco, L. Billeci, L. Ruta, S. Gangemi, G. Pioggia, Autism Research (2015)

27. A. Peca, M. Coeckelbergh, R. Simut, C. Costescu, S. Pintea, D. David, B. Vanderborght, IEEE Technology and Society Magazine 35(2), 54 (2016)

28. K. Dautenhahn, I. Werry, Proc. From animals to animats 6, 519 (2000)

29. M. Begum, R.W. Serna, H.A. Yanco, International Journal of Social Robotics 8(2), 157 (2016)

30. M. Coeckelbergh, C. Pop, R. Simut, A. Peca, S. Pintea, D. David, B. Vanderborght, Science and engineering ethics 22(1), 47 (2016)

31. F. Sartorato, L. Przybylowski, D.K. Sarko, Journal of psychiatric research 90, 1 (2017)

32. E. Broadbent, Annual review of psychology 68, 627 (2017)

33. X. Liu, Q. Wu, W. Zhao, X. Luo, Applied Sciences 7(10), 1051 (2017)

34. A.S. Weitlauf, N. Sathe, M.L. McPheeters, Z.E. Warren, Pediatrics 139(6), e20170347 (2017)

35. K. Dautenhahn, Robotica 21(04), 443 (2003)

36. J.J. Diehl, C.R. Crowell, M. Villano, K. Wier, K. Tang, L.D. Riek, in Comprehensive guide to autism (Springer, 2014), pp. 411-422

37. M.A. Miskam, S. Shamsuddin, M.R.A. Samat, H. Yussof, H.A. Ainudin, A.R. Omar, in MicroNanoMechatronics and Human Science (MHS), 2014 International Symposium on (IEEE, 2014), pp. $1-5$

38. M. Hirokawa, A. Funahashi, Y. Itoh, K. Suzuki, in The 23rd IEEE International Symposium on Robot and Human Interactive Communication (IEEE, 2014), pp. 365-370

39. I. Werry, K. Dautenhahn, W. Harwin, Procs AAATE 2001, (2001)

40. J. Lee, H. Takehashi, C. Nagai, G. Obinata, D. Stefanov, International Journal of Advanced Robotic Systems 9(3), 72 (2012)

41. J. Wainer, K. Dautenhahn, B. Robins, F. Amirabdollahian, International journal of social robotics 6(1), 45 (2014)
42. B. Huskens, A. Palmen, M. Van der Werff, T. Lourens, E. Barakova, Journal of autism and developmental disorders 45(11), 3746 (2015)

43. M.R.A. Samat, S. Shamsuddin, M.A. Miskam, H. Yussof, in Micro-NanoMechatronics and Human Science (MHS), 2014 International Symposium on (IEEE, 2014), pp. 1-5

44. M. Dimitrova, N. Vegt, E. Barakova, in Interactive Collaborative Learning (ICL), 2012 15th International Conference on (IEEE, 2012), pp. 1-8

45. P. Ponce, A. Molina, D. Grammatikou, Computers in Human Behavior 55, 28 (2016)

46. Y.D. Kim, J.W. Hong, W.S. Kang, S.S. Baek, H.S. Lee, J. An, in International Conference on Social Robotics (Springer, 2010), pp. 222-231

47. M.A. Ma'sum, M.S. Alvissalim, F. Sanjaya, W. Jatmiko, et al., in Advanced Computer Science and Information Systems (ICACSIS), 2012 International Conference on (IEEE, 2012), pp. 275-280

48. B. Robins, K. Dautenhahn, J. Dubowski, Interaction Studies 7(3), 509 (2006)

49. S.M. Anzalone, E. Tilmont, S. Boucenna, J. Xavier, A.L. Jouen, N. Bodeau, K. Maharatna, M. Chetouani, D. Cohen, M.S. Group, et al., Research in Autism Spectrum Disorders 8(7), 814 (2014)

50. N. Aresti-Bartolome, B. Garcia-Zapirain, BioMedical Materials and Engineering 26(s1), 811 (2015)

51. F. Petric, D. Tolić, D. Miklić, Z. Kovačić, M. Cepanec, S. Šimleša, in International Conference on Intelligent Robotics and Applications (Springer, 2015), pp. 82-94

52. Y. Pan, M. Hirokawa, K. Suzuki, in Robot and Human Interactive Communication (RO-MAN), 2015 24th IEEE International Symposium on (IEEE, 2015), pp. 48-53

53. B. Robins, in RO-MAN 200\%-The 16th IEEE International Symposium on Robot and Human Interactive Communication (IEEE, 2007), pp. 93-94

54. D. Conti, S. Di Nuovo, S. Buono, G. Trubia, A. Di Nuovo, in Robot and Human Interactive Communication (RO-MAN), 2015 24th IEEE International Symposium on (IEEE, 2015), pp. 1-6

55. D. Strickland, Presence: Teleoperators \& Virtual Environments 5(3), 319 (1996)

56. P. Chevalier, J.C. Martin, B. Isableu, C. Bazile, A. Tapus, Autonomous Robots 41(3), 613 (2017)

57. A. Steinfeld, T. Fong, D. Kaber, M. Lewis, J. Scholtz, A. Schultz, M. Goodrich, in Proceedings of the 1st ACM SIGCHI/SIGART conference on Human-robot interaction (ACM, 2006), pp. 33-40 
58. K. Dautenhahn, International Journal of Advanced Robotic Systems 4(1), 15 (2007)

59. M.A. Goodrich, A.C. Schultz, et al., Foundations and Trends $\mathrm{R}$ in Human-Computer Interaction 1(3), 203 (2008)

60. F. Amirabdollahian, B. Robins, K. Dautenhahn, Z. Ji, in 2011 Annual International Conference of the IEEE Engineering in Medicine and Biology Society (IEEE, 2011), pp. 5347-5351

61. H. Kozima, Y. Yasuda, C. Nakagawa, in ROMAN 2007-The 16th IEEE International Symposium on Robot and Human Interactive Communication (IEEE, 2007), pp. 599-604

62. E. Bekele, J.A. Crittendon, A. Swanson, N. Sarkar, Z.E. Warren, Autism 18(5), 598 (2014)

63. M.D. Manner, in Proceedings of the 24th International Conference on Artificial Intelligence (AAAI Press, 2015), pp. 4383-4384

64. E.I. Barakova, P. Bajracharya, M. Willemsen, T. Lourens, B. Huskens, Expert Systems 32(6), 698 (2015)

65. Z. Zheng, E.M. Young, A. Swanson, A. Weitlauf, Z. Warren, N. Sarkar, in Advanced Robotics (ICAR), 2015 International Conference on (IEEE, 2015), pp. 72-77

66. J.C. Gillesen, E. Barakova, B.E. Huskens, L.M. Feijs, in 2011 IEEE International Conference on Rehabilitation Robotics (IEEE, 2011), pp. 1-7

67. S. Shamsuddin, L.I. Ismail, H. Yussof, N.I. Zahari, S. Bahari, H. Hashim, A. Jaffar, in Control System, Computing and Engineering (ICCSCE), 2011 IEEE International Conference on (IEEE, 2011), pp. 511-516

68. B. Robins, K. Dautenhahn, International journal of social robotics $\mathbf{6}(3), 397$ (2014)

69. H. Kozima, C. Nakagawa, in in Mobile Robots towards New Applications, Aleksandar Lazinica, Ed., Vienna: Advanced Robotic Systems (2006), pp. $271-286$

70. B. Vanderborght, R. Simut, J. Saldien, C. Pop, A.S. Rusu, S. Pintea, D. Lefeber, D.O. David, Interaction Studies 13(3), 348 (2012)

71. M.J. Salvador, S. Silver, M.H. Mahoor, in 2015 IEEE International Conference on Robotics and Automation (ICRA) (IEEE, 2015), pp. 6128-6133

72. J. Kajopoulos, A.H.Y. Wong, A.W.C. Yuen, T.A. Dung, T.Y. Kee, A. Wykowska, in International Conference on Social Robotics (Springer, 2015), pp. 296-305

73. T. Salter, N. Davey, F. Michaud, in The 23rd IEEE International Symposium on Robot and Human Interactive Communication (IEEE, 2014), pp. $574-579$

74. B. Robins, K. Dautenhahn, in International Conference on Social Robotics (Springer, 2010), pp. $243-252$

75. H. Kozima, C. Nakagawa, Y. Yasuda, Progress in Brain Research 164, 385 (2007)

76. S. Shamsuddin, H. Yussof, S. Mohamed, F.A. Hanapiah, Procedia Computer Science 42, 9 (2014)

77. K. Dautenhahn, Philosophical Transactions of the Royal Society B: Biological Sciences 362(1480), 679 (2007)

78. C.M. Goulart, J. Castillo, C.T. Valadao, E. Caldeira, T.F. Bastos-Filho, in 2014 IEEE 23rd International Symposium on Industrial Electronics (ISIE) (IEEE, 2014), pp. 1555-1559

79. P.B.S.N.T.R.A.R.W.E.V.V.J.K.S.J.R.W.Z. Weitlauf AS, McPheeters ML, Therapies for Children With Autism Spectrum Disorder: Behavioral Interventions Update. Comparative Effectiveness Review No. 137 ((Prepared by the Vanderbilt Evidence-based Practice Center under Contract No. 290-2012-00009-I.) AHRQ Publication No. 14EHC036-EF. Rockville, MD: Agency for Healthcare Research and Quality, 2014)

80. S. Lindgren, A. Doobay, Evidence-based interventions for autism spectrum disorders (2011)

81. J. Greczek, E. Kaszubski, A. Atrash, M. Matarić, in The 23rd IEEE International Symposium on Robot and Human Interactive Communication (IEEE, 2014), pp. 561-566

82. E.T. Bekele, U. Lahiri, A.R. Swanson, J.A. Crittendon, Z.E. Warren, N. Sarkar, IEEE Transactions on Neural Systems and Rehabilitation Engineering 21(2), 289 (2013)

83. S. Costa, H. Lehmann, K. Dautenhahn, B. Robins, F. Soares, International journal of social robotics $7(2), 265$ (2015)

84. C.A. Costescu, B. Vanderborght, D.O. David, Journal of autism and developmental disorders 45(11), 3715 (2015)

85. G. Pioggia, R. Igliozzi, M. Ferro, A. Ahluwalia, F. Muratori, D. De Rossi, IEEE Transactions on Neural Systems and Rehabilitation Engineering 13(4), 507 (2005)

86. G. Pioggia, M. Sica, M. Ferro, R. Igliozzi, F. Muratori, A. Ahluwalia, D. De Rossi, in RO-MAN 2007-The 16th IEEE International Symposium on Robot and Human Interactive Communication (IEEE, 2007), pp. 605-612

87. R. Simut, C. Pop, B. Vanderborght, J. Saldien, A. Rusu, S. Pintea, J. Vanderfaeillie, D. Lefeber, D. David, et al., in 3rd International conference on 
Social Robotics (ICSR 2011) (2011), pp. 97-100

88. L. Boccanfuso, J.M. O'Kane, International journal of social robotics $\mathbf{3}(4), 337$ (2011)

89. S. Shamsuddin, H. Yussof, L.I. Ismail, S. Mohamed, F.A. Hanapiah, N.I. Zahari, Procedia Engineering 41, 1448 (2012)

90. R.S. Moorthy, S. Pugazhenthi, International Journal of Social Robotics 9(1), 97 (2017)

91. H. Kumazaki, Z. Warren, T. Muramatsu, Y. Yoshikawa, Y. Matsumoto, M. Miyao, M. Nakano, S. Mizushima, Y. Wakita, H. Ishiguro, et al., PloS one 12(10), e0186581 (2017)

92. L.I. Ismail, H. Yussof, S. Shamsuddin, F.A. Hanapiah, N.I. Zahari, in Proceedings of the 7th International Convention on Rehabilitation Engineering and Assistive Technology (Singapore Therapeutic, Assistive \& Rehabilitative Technologies (START) Centre, 2013), p. 64

93. C.A. Pop, R. Simut, S. Pintea, J. Saldien, A. Rusu, D. David, J. Vanderfaeillie, D. Lefeber, B. Vanderborght, International Journal of Humanoid Robotics 10(03), 1350025 (2013)

94. H. Kozima, C. Nakagawa, Y. Yasuda, in ROMAN 2005. IEEE International Workshop on Robot and Human Interactive Communication, 2005. (IEEE, 2005), pp. 341-346

95. J. Wainer, B. Robins, F. Amirabdollahian, K. Dautenhahn, IEEE Transactions on Autonomous Mental Development 6(3), 183 (2014)

96. M.S. Erden, International Journal of Social Robotics 5(4), 441 (2013)

97. A. Tapus, A. Peca, A. Aly, C. Pop, L. Jisa, S. Pintea, A.S. Rusu, D.O. David, Interaction studies 13(3), 315 (2012)

98. L. Dickstein-Fischer, E. Alexander, X. Yan, H. Su, K. Harrington, G.S. Fischer, in 2011 Annual International Conference of the IEEE Engineering in Medicine and Biology Society (IEEE, 2011), pp. $5319-5322$

99. E.S. Kim, L.D. Berkovits, E.P. Bernier, D. Leyzberg, F. Shic, R. Paul, B. Scassellati, Journal of autism and developmental disorders 43(5), 1038 (2013)

100. D. Feil-Seifer, M.J. Matarić, in Experimental robotics (Springer, 2009), pp. 201-210

101. E.S. Kim, C.M. Daniell, C. Makar, J. Elia, B. Scassellati, F. Shic, in Affective Computing and Intelligent Interaction (ACII), 2015 International Conference on (IEEE, 2015), pp. 8-13

102. K.C. Welch, U. Lahiri, Z. Warren, N. Sarkar, International journal of social robotics 2(4), 391 (2010)
103. M.G. Kim, E. Barakova, T. Lourens, in The 23rd IEEE International Symposium on Robot and $\mathrm{Hu}$ man Interactive Communication (IEEE, 2014), pp. 353-358

104. S.S. Yun, S.K. Park, J. Choi, in The 23rd IEEE International Symposium on Robot and Human Interactive Communication (IEEE, 2014), pp. 130134

105. E. Delaherche, S. Boucenna, M. Chetouani, D. Cohen, in Neural Nets and Surroundings (Springer, 2013), pp. 345-356

106. I.B. Wijayasinghe, I. Ranatunga, N. Balakrishnan, N. Bugnariu, D.O. Popa, International Journal of Social Robotics 8(5), 695 (2016)

107. S. Shamsuddin, H. Yussof, F. Hanapiah, S. Mohamed, Proceedings of Mechanical Engineering Research Day 2015: MERD'15 2015, 145 (2015)

108. M. Kim, T. Kwon, K. Kim, International Journal of Social Robotics 10(1), 33 (2018)

109. M. de Haas, I. Smeekens, E. Njeri, P. Haselager, J. Buitelaar, T. Lourens, W. Staal, J. Glennon, E. Barakova, in Robotics in Education (Springer, 2017), pp. 259-270

110. O. Rudovic, J. Lee, L. Mascarell-Maricic, B.W. Schuller, R.W. Picard, Frontiers in Robotics and AI 4, 36 (2017)

111. M. Hirokawa, A. Funahashi, Y. Pan, Y. Itoh, K. Suzuki, in Robot and Human Interactive Communication (RO-MAN), 2016 25th IEEE International Symposium on (IEEE, 2016), pp. 843-848

112. R. Suzuki, J. Lee, in Micro-NanoMechatronics and Human Science (MHS), 2016 International Symposium on (IEEE, 2016), pp. 1-5

113. P. Chevalier, J.C. Martin, B. Isableu, C. Bazile, D.O. Iacob, A. Tapus, in Robot and Human Interactive Communication (RO-MAN), 2016 25th IEEE International Symposium on (IEEE, 2016), pp. 849-854

114. J.M. Beer, M. Boren, K.R. Liles, in Human-Robot Interaction (HRI), 2016 11th ACM/IEEE International Conference on (IEEE, 2016), pp. 419-420

115. X. Liu, X. Zhou, C. Liu, J. Wang, X. Zhou, N. Xu, A. Jiang, in Human System Interactions (HSI), 2016 9th International Conference on (IEEE, 2016), pp. 56-61

116. Z.E. Warren, Z. Zheng, A.R. Swanson, E. Bekele, L. Zhang, J.A. Crittendon, A.F. Weitlauf, N. Sarkar, Journal of autism and developmental disorders 45(11), 3726 (2015)

117. C. Huijnen, M. Lexis, L. de Witte, Int J Neurorehabilitation 4(278), 2376 (2017)

118. C.A. Huijnen, M.A. Lexis, L.P. de Witte, International Journal of Social Robotics 8(4), 445 (2016) 
119. V. Silva, F. Soares, J.S. Esteves, in Bioengineering (ENBENG), 2017 IEEE 5th Portuguese Meeting on (IEEE, 2017), pp. 1-4

120. J. Bharatharaj, L. Huang, A. Al-Jumaily, R.E. Mohan, C. Krägeloh, International Journal of Advanced Robotic Systems 14(5), 1729881417736895 (2017)

121. J. Bharatharaj, L. Huang, A. Al-Jumaily, M.R. Elara, C. Krägeloh, in IOP Conference Series: Materials Science and Engineering, vol. 234 (IOP Publishing, 2017), vol. 234, pp. 012-017

122. S.S. Yun, J. Choi, S.K. Park, G.Y. Bong, H. Yoo, Autism Research 10(7), 1306 (2017)

123. A. Guneysu, R.D. Siyli, A.A. Salah, in The 23rd IEEE International Symposium on Robot and Human Interactive Communication (IEEE, 2014), pp. 199-204

124. M. Begum, R.W. Serna, D. Kontak, J. Allspaw, J. Kuczynski, H.A. Yanco, J. Suarez, in Proceedings of the Tenth Annual ACM/IEEE International Conference on Human-Robot Interaction (ACM, 2015), pp. 335-342

125. H.L. Cao, C. Pop, R. Simut, R. Furnemónt, A. De Beir, G. Van de Perre, P.G. Esteban, D. Lefeber, B. Vanderborght, in International Conference on Social Robotics (Springer, 2015), pp. $93-102$

126. M.C. Chuah, D. Coombe, C. Garman, C. Guerrero, J. Spletzer, in 2014 IEEE 11th International Conference on Mobile Ad Hoc and Sensor Systems (IEEE, 2014), pp. 731-736

127. S. Shamsuddin, H. Yussof, L. Ismail, F.A. Hanapiah, S. Mohamed, H.A. Piah, N.I. Zahari, in Signal Processing and its Applications (CSPA), 2012 IEEE 8th International Colloquium on (IEEE, 2012), pp. 188-193

128. C. Vandevelde, J. Saldien, in Human-Robot Interaction (HRI), 2016 11th ACM/IEEE International Conference on (IEEE, 2016), pp. 555-556 